\title{
Load Balancing Network by using Round Robin Algorithm: A Systematic Literature Review
}

\author{
Taufik Hidayat $^{1}$, Yasep Azzery ${ }^{2}$, Rahutomo Mahardiko ${ }^{3}$ \\ ${ }^{1}$ Department of Computer Engineering, Universitas Wiralodra, Indramayu, Indonesia \\ ${ }^{2}$ Department of Electrical Engineering, Universitas Mercu Buana, Jakarta, Indonesia \\ ${ }^{3}$ Platinumetrix Pte. Ltd, Jakarta, Indonesia \\ 1'thidayat.ft@unwir.ac.id, ${ }^{2}$ yasep.azzery@gmail.com, ${ }^{3}$ rahutomo.mahardiko@gmail.com
}

\begin{abstract}
The use of load balance on a network will be very much needed if the network is an active network and is widely accessed by users. A reason is that it allows network imbalances to occur. Round Robin (RR) algorithm can be applied for network load balancing because it is a simple algorithm to schedule processes so that it can provide work process efficiency. Authors use the Systematic Literature Review (SLR) method in which it can be applied for criteria selection during papers search to match the title being raised. SLR is divided into five stages, namely formalization of questions, criteria selection, selection of sources, selection of search results, and quality assessment. By using SLR, it is expected that papers according to criteria and quality can be found.
\end{abstract}

Keywords- Load Balance, Round Robin Algorithm, Security, Network, Systematic Literature Review

\section{INTRODUCTION}

Security in a network is one thing that must be ensured by every user, especially internet users. Router is one of the network devices that has functions as a liaison between nodes in the network. In a static sensor network, the settings and distribution of sensor nodes are completed during the network initialization process. After either random or location-based deployment, each node maintains its position, then conveys sensing data [1].

The RR algorithm is one of the simplest load balancing algorithms used lately. It uses a circular list and a pointer to the last selected server to make a shipping decision. It sends user requests to web server from first web server until the last one with command [2]. The authors discuss load balancing on router devices with the RR method.

By utilizing SLR, writers discuss load balancing in networks with the RR method. Scheduling system in the algorithm uses a waiting time scheme that all data will be processed sequentially according to the scheduling process [3]. Researchers found various papers and studies on load balances and RR as contained in table 1 which is become a reference to use SLR. The rest of paper will contain related work, research method, discussion and result, and conclusions and future work.

Table 1. Online Library

\begin{tabular}{ccc}
\hline No. & $\begin{array}{c}\text { Digital Online } \\
\text { Library }\end{array}$ & Website \\
\hline 1. & IEEE Explore & ieeexplore.ieee.org \\
2. & ScienceDirect & www.sciencedirect.com
\end{tabular}

A. Round Robin Algorithm
Load balancing technique with RR algorithm is one of the most classic techniques and is still widely used. The technique is very simple. Concept is to distribute client requests across servers [4]. The RR load balancer forwards the client requests to each server one at a time [5]. After reaching the end of the list of available servers, then it goes back and starts setting client requests again from the first server. The main advantage of this algorithm is that it is very easy to implement. But in a scenario where load requests differ significantly, it cannot distribute loads efficiently [6].

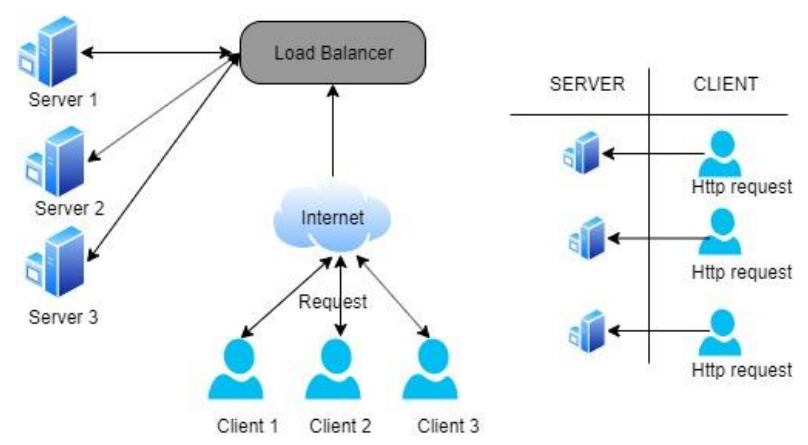

Figure 1. Infrastructure RR Algorithm [7]

Each connection has different service time. When the time required is too long, the connection will be disconnected and logged out from the network. In the RR Based Smart Priority algorithm, Time Slice (ITS) has been estimated and allocated different processing times for each service according to their assignment of priority basis. The method focuses on measurements that have different 
parameters from the RR algorithm. The following parameters are [8].

\section{Burst Time (BT)}

BT is the time needed to complete the execution of certain service requests.

2. Time Quantum (TQ)

TQ is the period of time given to allow a service to access a VM.

Monitoring on the RR algorithm allows the load balancer to distinguish between several services on a single server as if one service is not available. Other services on the same server might work to accept loads with a RR scheme. This algorithm uses basics such as the number of current connections, host utilization, and even realtime response times for existing traffic to the server to choose the appropriate server from the available cluster services [9].

\section{B. Load Balance}

Any high load traffic in a network is that often causes problems in certain network ranges. So, there is a need to be aware of its configuration. In a computer network on the internet, a connected computer will be given a public Internet Protocol (IP) address. Before a connected computer join the internet, it only has a private IP address. There is only one public IP address given by an Internet Service Provider (ISP). Sometimes many users have the same public IP address [8].

In the use of load balance on a network, it will be very necessary if the network is an active network and is accessed by many users. Load balance happens because it allows network imbalances [10]. Load balance is a configuration way to manage load similarity to be more stable by using less ISP lines used or active or rather merging ISP lines. The purpose of load balancing is to increase acceptance, to overcome if there is a system failure, to maintain system strength, and to manage the reduction upcoming traffic [1].

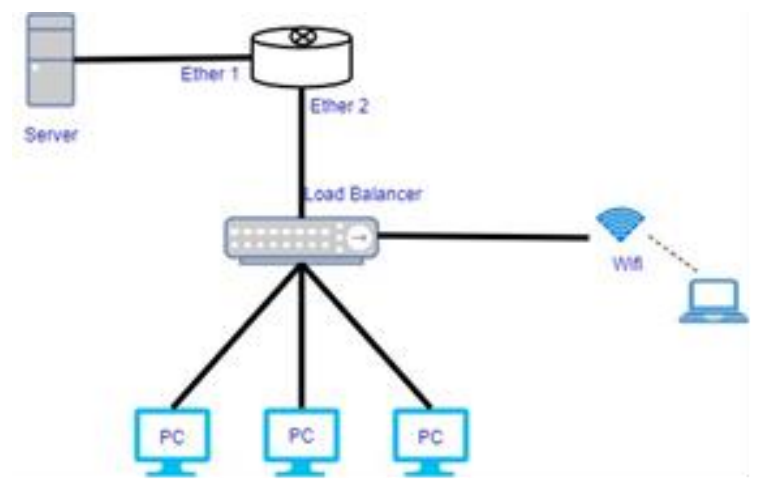

Figure 2. Infrastructure Load Balancer[7]
Load balancers are generally installed between the client and the server, which provide the services needed by the client and other similar arrangements for load balancing. In this work scenario, the load balance can also communicate with other people to return to load balancer point to process the return path to the client. The load balancing process depends on network characteristics, such as: checking for lost packets, delaying or monitoring connection conditions [9], [11].

\section{METHOD}

Authors use SLR research method to understand the application of RR in the load balancing network. In this SLR, there are five stages. First stage is formalization of questions. Second stage is search for criteria. Third stage is selection of sources. Fourth stage is selection of search results. Last stage is quality assessment [12]. The SLR method also aims to survey the mechanism used in other paper or to compare search results from other search sites [13].

Table 1 is two trusted search engine (IEEE Explore and Sciencedirect) in order to search relevant paper starting from 2015 to 2019. From search results based on these sites, the authors hope to find a paper that has good quality according to several selection processes [12].

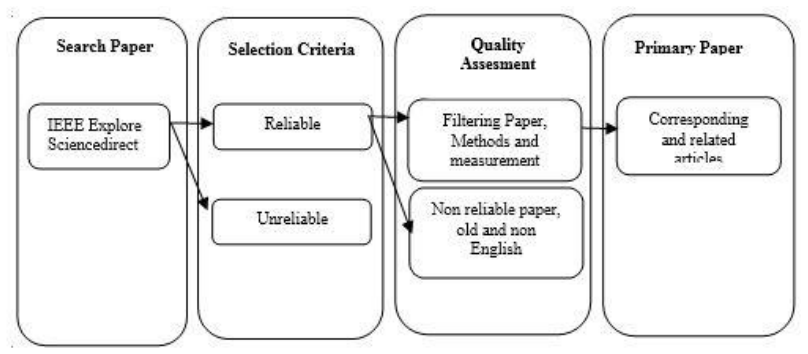

Figure 3. Article Identification Process [9]

The authors conducted various stages of identifying the paper by using two digital library sites with various stages. First stage is selection criteria. This stage is to make an initial selection of whether the paper is still related to the title of author's paper and related to the method used. If the paper is related to author's theme, then filter on paper, method, and measurements. After that, it is a need to make sure that those papers are appropriate and acceptable. If the obtained results of the paper selection does not find English paper, then the paper cannot be used as a reference. After all filtering and measurement processes are well complete, a paper will be obtained in accordance with the conditions set by the researcher and become the basis for further research.

The sequence of processes in figure 3 shows the SLR scheme in finding relevant paper according to specified criteria and for finding high quality paper. 
Table 2. Criteria of Paper SLR

\begin{tabular}{|c|c|}
\hline ID & Unreliable \\
\hline U1 & $\begin{array}{l}\text { The focus of the paper has not been updated } \\
\text { with the current conditions }\end{array}$ \\
\hline $\mathrm{U} 2$ & The paper uses language other than English \\
\hline \multirow[t]{2}{*}{ U3 } & $\begin{array}{l}\text { Paper discussion material is not very related to } \\
\text { what is sought by researchers }\end{array}$ \\
\hline & Reliable \\
\hline R1 & $\begin{array}{l}\text { The contents of the paper are still up to date } \\
\text { with the current conditions }\end{array}$ \\
\hline $\mathrm{R} 2$ & $\begin{array}{l}\text { Paper that discusses the concept of load } \\
\text { balance }\end{array}$ \\
\hline R3 & Paper that discusses the RR algorithm \\
\hline \multirow[t]{2}{*}{$\mathrm{R} 4$} & Paper published from 2015 to 2019 \\
\hline & Quality Assesment \\
\hline QA1 & What methods are used in related Papers? \\
\hline QA2 & What measuring instruments are used? \\
\hline QA3 & $\begin{array}{l}\text { What are the measurement results with the } \\
\text { method used? }\end{array}$ \\
\hline QA4 & $\begin{array}{l}\text { Do researchers refer to research that has } \\
\text { already existed? }\end{array}$ \\
\hline QA5 & How are the improvements made? \\
\hline QA6 & $\begin{array}{l}\text { Are the results of applying the method with the } \\
\text { object discussed in accordance with the criteria } \\
\text { of the researcher? }\end{array}$ \\
\hline
\end{tabular}

Based on table 2 on the criteria of paper selection with SLR and reliability determined by authors, several papers were found in accordance with the criteria based on the provisions and questions from the table. Those papers are then the results related to the title "Load Balancing Network by Using Round Robin Algorithm: A Systematic Literature Review". The process was done by using trusted paper database in the online library of IEEE Explore and Sciencedirect. Authors used filter between 2015 and 2019. The search was conducted on October 5, 2019. The results of found paper that were searched from two online library sites can be seen in the following figure.

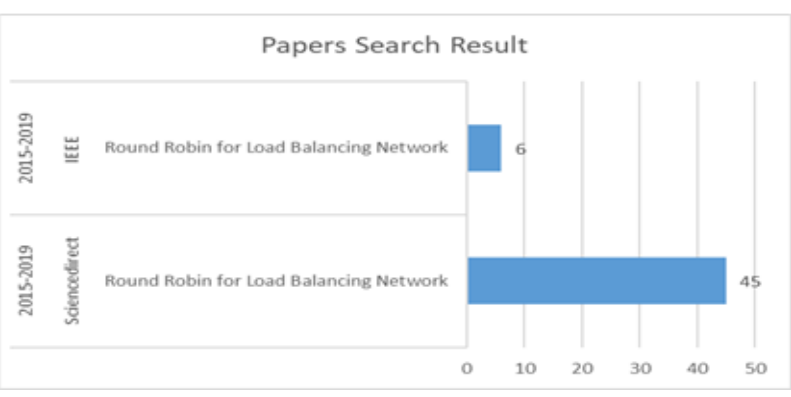

Figure 4. Papers search results

Figure 4 shows that there are 51 papers associated with the title. Those papers were retrieved from two digital library sites that did not use the SLR method. From the results of the paper search, the authors want to develop the title into SLR format so that the paper can develop with other methods and produce other quality papers about the application of the RR algorithm in the load balancing network.

\section{RESULT AND DISCUSSION}

\section{A. Load Balance Network}

As of the papers that have been sought by researchers based on established criteria and stages, there are 6 papers from IEEE Explore and 45 papers from Sciencedirect as a reference to be researched. Load balancing system which is one of the methods to regulate traffic balance on a network is a matter important [10]. The RR method for scheduling is one of the simplest method that can be used in network load balancing [2]. Load balancing ensures that each node has the same number of nodes so that the balance between nodes can be maintained [14]. Authors found 2 papers related to SLR load balance from digital library and there is no paper regarding load balance network with RR algorithm.

\section{B. SLR Journal in Online Library Screening}

Researchers used two online library databases and screened them according to the filtered titles from 2015 to 2019. The target is to get relevant papers and the papers are still up to date with current conditions.

Table 3. Screening Result of SLR for Papers in Database

\begin{tabular}{|c|c|c|c|}
\hline Year & Database & Keyword & Result \\
\hline $\begin{array}{l}2015- \\
2019\end{array}$ & Sciencedirect & Load Balancing & 173217 \\
\hline 2015- & & Load Balancing & 62114 \\
\hline 2019 & & Network & \\
\hline 2019 & & $\begin{array}{l}\text { Round Robin for Load } \\
\text { Balancing Network }\end{array}$ & 1 \\
\hline $\begin{array}{l}2015- \\
2019\end{array}$ & IEEE & Load Balancing & 2139 \\
\hline $\begin{array}{l}2015- \\
2019\end{array}$ & & $\begin{array}{l}\text { Load Balancing } \\
\text { Network }\end{array}$ & 1073 \\
\hline 2019 & & $\begin{array}{l}\text { Round Robin for Load } \\
\text { Balancing Network }\end{array}$ & 2 \\
\hline
\end{tabular}

Based on the search data in table 3, authors found an interesting thing that Sciencedirect site has the most paper in load balancing. For the RR keyword for load balancing network, researchers found 1 paper in Sciencedirect and 2 papers in IEEE. Based on the data of the search results, researchers took relevant papers in accordance with the theme raised about the use of the RR algorithm on the load balancing network. In this case, by using RR, the load balance network technique becomes more efficient and effective in terms of process, time and cost. The use of load balance with the RR algorithm is still not widely applied. So, researchers are interested in finding more in-depth information about the effect of the RR algorithm on the load balance technique with SLR method to produce more quality paper.

Figure 5 is the results of the papers from database selection activity. The results are then considered to have the same method with author paper and also discuss the load balancing network with the RR algorithm. 


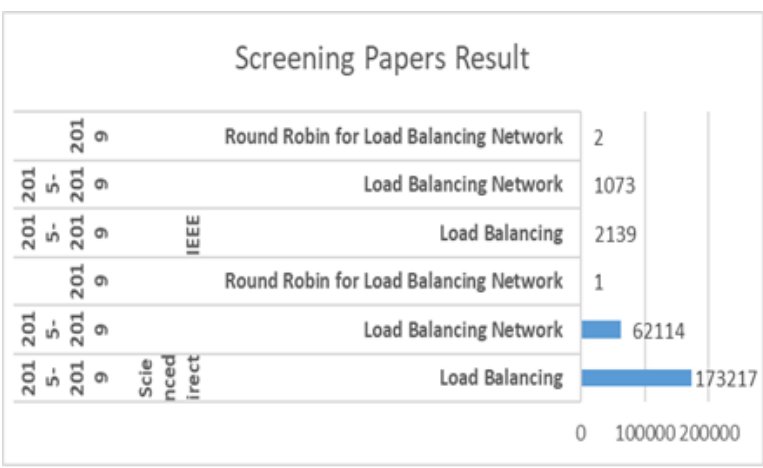

Figure 5. Database Screening Results Graph

From the results of the screening journal database from 2015 to 2019, authors found that little paper which discussed network load balancing with the RR algorithm. There are only 2 journals from the IEEE Explore site and 1 journal from Sciencedirect. Currently, research on load balance uses several algorithms for solving load balances. However, the use of RR, one of the simplest algorithm, can be used for load balancing techniques [2]. The selection criteria activity only found 1 journal that was more focused on discussion about the use of the RR algorithm for wireless load balancing [15].

\section{IV.CONCLUSION}

RR algorithm can be applied to network load balancing because it is a simple algorithm in scheduling processes. So, it can provide work process efficiently. According to RR predictions, load balancers can provide shipping decisions when the server is selected to process user requests and time efficiency.

In accordance to networks on a large scale, it is a need to keep load record on each server node. So we can know in outline the process description, capacity usage and the amount of web server load. In this case, we can even have an overall assessment on the cost and performance of the server. The screening data process shows that there are 3 journals discussing load balancing with the RR algorithm. No journal has focused on load balancing networks discussion with the RR method.

Authors found only a few papers using the SLR method. In the future, there are still many researches that can be explored and developed again, especially about load balancing networks with the RR algorithm. Because this algorithm is simple in its technique and is efficient of time and work processes.

\section{ACKNOWLEDGEMENTS}

The authors would like to thank Department of Computer Engineering, Universitas Wiralodra and Department of Electrical Engineering, Universitas Mercu Buana for support of this research.

\section{REFERENCES}

[1] S. Kaur and T. Sharma, "Efficient load balancing using improved central load balancing technique," Proc. 2nd Int. Conf. Inven. Syst. Control. ICISC 2018, no. Icisc, pp. 1-5, 2018.

[2] Z. Xu and X. Wang, "A predictive modified round robin scheduling algorithm for web server clusters," Chinese Control Conf. CCC, vol. 2015Septe, pp. 5804-5808, 2015.

[3] M. A. Mohammed, M. Abdulmajid, B. A. Mustafa, and R. F. Ghani, "Queueing theory study of round robin versus priority dynamic quantum time round robin scheduling algorithms," 2015 4th Int. Conf. Softw. Eng. Comput. Syst. ICSECS 2015 Virtuous Softw. Solut. Big Data, pp. 189-194, 2015.

[4] A. Erzurumluoğlu, "Constructing day-balanced round-robin tournaments with partitions," Discret. Appl. Math., vol. 235, pp. 81-91, 2018.

[5] S. Abdallah, E. Najjar, and A. Kayssi, "A round robin load balancing and redundancy protocol for network routers," Proc. 11 th IEEE Int. Conf. Trust. Secur. Priv. Comput. Commun. Trust. - 11th IEEE Int. Conf. Ubiquitous Comput. Commun. IUCC2012, pp. 1741-1747, 2012.

[6] L. Wang and G. Lu, "The Dynamic Sub-Topology Load Ba lancing Algorithm for Data Center Networks," 2016 Int. Conf. Inf. Netw., pp. 268273, 2016.

[7] Q. Shi, F. Wang, D. Feng, and W. Xie, "Adaptive load balancing based on accurate congestion feedback for asymmetric topologies," Comput. Networks, vol. 157, pp. 133-145, 2019.

[8] S. Ghosh and C. Banerjee, "Dynamic time quantum priority based round robin for load balancing in cloud environment," Proc. - 2018 4th IEEE Int. Conf. Res. Comput. Intell. Commun. Networks, ICRCICN 2018, pp. 33-37, 2019.

[9] M. H. Raza, A. Nafarieh, and W. Robertson, "Application of network tomography in load balancing," Procedia Comput. Sci., vol. 52, no. 1, pp. 1120-1125, 2015.

[10] Q. Huang, J. Tan, and W. Jiang, "A new load balancing routing scheme for wireless body area networks," Proc. 2019 IEEE 3rd Inf. Technol. Networking, Electron. Autom. Control Conf. ITNEC 2019, no. Itnec, pp. 866-870, 2019.

[11] L. Cheng, S. Kotoulas, Q. Liu, and Y. Wang, "Load-balancing distributed outer joins through operator decomposition," J. Parallel Distrib. Comput., vol. 132, pp. 21-35, 2019.hhahe

[12] A. S. Milani and N. J. Navimipour, "Load balancing mechanisms and techniques in the cloud environments: Systematic literature review and future trends," J. Netw. Comput. Appl., vol. 71, pp. 86-98, 2016.

[13] A. A. Neghabi, N. J. Navimipour, M. Hosseinzadeh, and A. Rezaee, "Load Balancing Mechanisms in the Software Defined Networks: A 
Systematic and Comprehensive Review of the Literature," IEEE Access, vol. 6, no. c, pp. 1415914178, 2018.

[14] J. C. Patni and M. S. Aswal, "Distributed load balancing model for grid computing environment," Proc. 2015 1st Int. Conf. Next Gener. Comput. Technol. NGCT 2015, no. September, pp. 123126, 2016.

[15] E. F. Aza and J. P. Urrea, "Implementation of Round-Robin load balancing scheme in a wireless software defined network," 2019 IEEE Colomb. Conf. Commun. Comput., pp. 1-6, 2019. 